\title{
Experimental Study of Magnetic Properties of Alumina Ceramics
}

\author{
R. Tarasenko*, A. OrendáČová, M. OrendáČ, M. Kajňaková and A. Feher \\ Centre of Low Temperature Physics of SAS and P.J. Šafárik University \\ Park Angelinum 9, 04154 Košice, Slovak Republic
}

\begin{abstract}
Magnetic properties of alumina ceramics were studied within a program focused at design of microcalorimeters and experimental setup for specific heat and magnetocaloric measurements. The investigations were concentrated on the determination of amount of magnetic impurities that can significantly influence results of physical measurements at low temperatures. Our experimental studies of heat capacity, susceptibility and magnetization clearly indicate that alumina ceramics contains magnetic impurities, primarily ions with spin $5 / 2$. The average concentration of magnetic ions was estimated, $n \approx 2000 \mathrm{ppm}$. More details of analysis of experimental data are discussed in the paper.
\end{abstract}

PACS numbers: 75.20.-g, 75.60.Ej, 75.40.-s

\section{Introduction}

Many different materials are used for low temperature calorimetric experiments. When designing experimental setup, attention must be focused at thermal conductivity and heat capacity - quantities which affect the timescale of experiment. Crystalline alumina (chemical formula $\mathrm{Al}_{2} \mathrm{O}_{3}$ ) is a frequently used material due to relatively high thermal conductivity, low specific heat and high hardness. On the other hand, alumina ceramics changes thermal conductivity within 3 orders of magnitude in the temperature range from 0.1 to $1 \mathrm{~K}$ [1]. Due to this property and high hardness, it can be promising material for construction of a sample holder in magnetocaloric experiments. However, commercially prepared alumina ceramics [2] can contain various amount of magnetic impurities, which can contribute to undesirable effects. This work deals with the magnetic properties of white alumina ceramics with the aim to clarify the influence of magnetic impurities on the thermodynamic properties at low temperatures.

\section{Experimental details}

The sample of white alumina ceramics in the form of a plate of weight $230 \mathrm{mg}$ was used for specific heat measurement. The specific heat data were collected using a dual slope method in a commercial dilution ${ }^{3} \mathrm{He}-{ }^{4} \mathrm{He}$ refrigerator TLE 200. The copper foil of weight $45 \mathrm{mg}$

* corresponding author; e-mail:

robert.tarasenko@student.upjs.sk was mounted between the sample and the home-made microcalorimeter for better thermal contact. The static magnetic susceptibility and magnetization measurements were performed in a commercial SQUID magnetometer on the sample weighing $350 \mathrm{mg}$.

\section{Results and discussion}

The specific heat studies were performed in the temperature range from $90 \mathrm{mK}$ to $1.4 \mathrm{~K}$ in zero magnetic field. The behaviour of the data is characterized by a round maximum of the Schottky-type observed at $T_{\mathrm{MAX}} \approx 0.5 \mathrm{~K}$ and a monotonic increase is observed at higher temperatures (Fig. 1, bottom inset). The observed low value of the temperature $T_{\text {MAX }}$ can be associated with the presence of weak crystal field splitting arising from magnetic impurities, which is typical for ions with $S \geq 1$. Previous specific heat studies of commercial thick film ruthenate resistors have revealed the presence of $\mathrm{Fe}^{3+}$ ion impurities with $S=5 / 2$ in alumina substrate with the concentration $\approx 500 \mathrm{ppm}[3]$. Correspondingly, in our analysis, magnetic impurities were identified as $\mathrm{Fe}^{3+}$ ions with $S=5 / 2$. Because the sample represents a magnetic insulator, the total specific heat, $C_{\mathrm{TOT}}$, is determined by the sum of the magnetic contribution, $C_{\text {mag }}$ and phonon subsystem $C_{\mathrm{ph}}$. $C_{\mathrm{TOT}}$ can be described in some temperature interval with a formula $C_{\mathrm{TOT}}=C_{\mathrm{mag}}+C_{\mathrm{ph}}=a / T^{2}+b T^{3}$. A linear fit of the $C T^{2}$ vs. $T^{5}$ has been performed in the range $1.2-1.4 \mathrm{~K}$ providing $a=2.25 \times 10^{-5} \mathrm{~J} \mathrm{~K}$ and $b=6.84 \times 10^{-6} \mathrm{~J} \mathrm{~K}^{-4}$ (Fig. 1, top inset). After subtracting the lattice contribution, the temperature dependence of magnetic heat capacity was compared with a theoretical prediction for a simple model of a spin $5 / 2$ paramagnet with crystal 


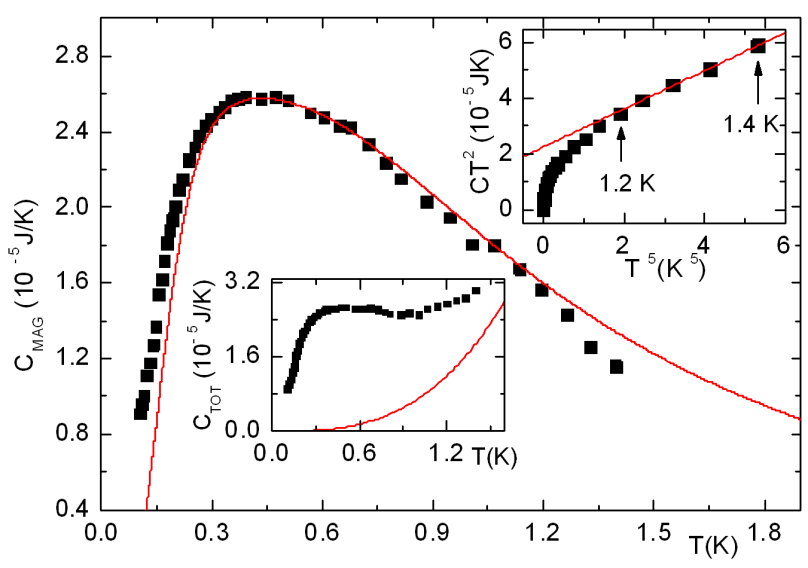

Fig. 1. Temperature dependence of magnetic heat capacity of alumina ceramics (squares). The solid line represents the $S=5 / 2$ paramagnet with $D / k_{\mathrm{B}}=0.37 \mathrm{~K}$ and concentration of $\mathrm{Fe}^{3+}$ ions 2700 ppm. Bottom inset: temperature dependence of total alumina heat capacity in zero magnetic field (squares). The lattice contribution in the Debye approximation is presented by solid line. Top inset: $C T^{2}$ vs. $T^{5}$ (squares), solid line represents the fit $y=a+b x$ with $x=T^{5}, y=C T^{2}$. The arrows indicate the fitting interval.

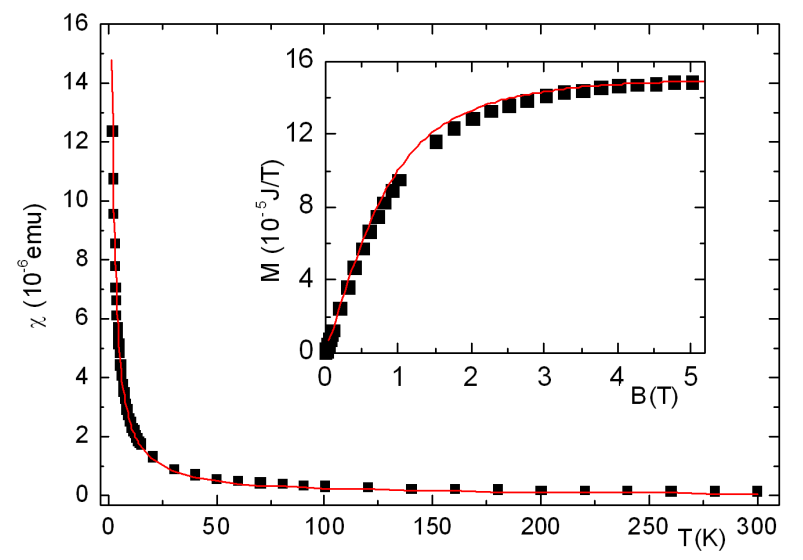

Fig. 2. Temperature dependence of the magnetic susceptibility of alumina ceramics measured in the magnetic field of $100 \mathrm{mT}$ (squares). The solid line represents the theoretical prediction of susceptibility of powdered sample for the paramagnetic system with $S=5 / 2$, $g=2, D / k_{\mathrm{B}}=0.37 \mathrm{~K}$ and concentration of iron $n=2050 \mathrm{ppm}$. Inset: magnetic field dependence of magnetization of alumina at the constant temperature $1.8 \mathrm{~K}$ (squares). Ideal, free-spin behaviour described by the Brillouin function with $g=2, S=5 / 2$ and $n=1570 \mathrm{ppm}$ is presented by the solid line.

field splitting represented by single-ion anisotropy constant $D[4]$. The best agreement was found for the model with $D / k_{\mathrm{B}}=0.37 \mathrm{~K}$ and the concentration of $\mathrm{Fe}^{3+}$ ions was estimated, $n \approx 2700 \pm 300$ ppm (Fig. 1).

The susceptibility was measured in the temperature range from 1.8 to $300 \mathrm{~K}$ in magnetic field $100 \mathrm{mT}$ (Fig. 2). It should be noted that the measurements performed dur- ing field cooling and zero-field cooling yielded the same experimental results. The susceptibility has been corrected for the diamagnetic contribution of alumina ceramics, $\chi_{\text {diam }} \approx-4.8 \times 10^{-5} \mathrm{emu}$, which was estimated by using Pascal's constants. The data are well characterized by the Curie-Weiss law. Fitting the experimental data in the temperature interval from 6 to $40 \mathrm{~K}$ yielded the Curie constant $C=2.97 \times 10^{-5}$ emu K providing $n \approx 1970 \pm 200 \mathrm{ppm}$ and the Curie temperature $\Theta_{\mathrm{c}} \approx-1.74 \mathrm{~K}$. This value reflects the weak crystal field splitting as observed in specific heat. The temperature dependence of magnetic susceptibility was fitted with numerical prediction for a spin $5 / 2$ paramagnet with single-ion anisotropy constant $D / k_{\mathrm{B}}=0.37 \mathrm{~K}$ obtained from specific heat capacity (Fig. 2). This procedure yielded the concentration of iron $2050 \pm 200 \mathrm{ppm}$, which is in good agreement with the values obtained from the Curie constant and specific heat studies.

The magnetic field dependence of magnetization (Fig. 2, inset) has been studied at the constant temperature $1.8 \mathrm{~K}$ in magnetic fields up to $5 \mathrm{~T}$. The experimental data are well consistent with ideal, free-spin behaviour described by the Brillouin function with $g=2, S=5 / 2$ and the concentration of $\mathrm{Fe}^{3+}$ ions $1570 \pm 200 \mathrm{ppm}$.

\section{Conclusion}

Our experimental studies of heat capacity, susceptibility and magnetization clearly indicate that white alumina ceramics contains magnetic impurities, primarily ions with spin $5 / 2$. The average concentration of the ions was estimated as $n \approx 2000 \mathrm{ppm}$. Considering potential use of the alumina ceramics as a sample holder in specific heat studies, commercially produced materials are not suitable due to high concentration of magnetic impurities, which contribute to addenda even in zero magnetic field. In the near future, further experiments of magnetocaloric effect will be performed on alumina ceramics to find out the influence of the magnetic impurities on the temperature of alumina ceramics under semiadiabatic conditions.

\section{Acknowledgments}

This work was supported by project LPP-0202-09, VEGA grant No. 1/0078/09, project APVV-0006-07 and ERDF EU project No. ITMS26220120005. Material support from U.S. Steel Košice s.r.o. is greatly acknowledged.

\section{References}

[1] F. Pobell, Matter and Methods at Low Temperatures, Springer-Verlag, New York 1992.

[2] http://www.dynacer.com .

[3] Ya.E. Volokitin, R.C. Thiel, L.J. de Jongh, Cryogenics 34, 771 (1994).

[4] R. Boča, Coord. Chem. Rev. 248, 757 (2004). 